\title{
PLASMA EQUILIBRIUM IN TOKAMAKS
}

\author{
H.J. de Blank \\ FOM Institute DIFFER - Dutch Institute for Fundamental Energy Research, \\ Association EURATOM-FOM, P.O. Box 1207, 3430 BE Nieuwegein, The Netherlands, www. differ.nl.
}

\begin{abstract}
This lecture treats the magnetohydrodynamic (MHD) equilibrium of axisymmetric plasmas, as given by the GradShafranov equation. In a brief introduction, equilibrium parameters such as the $q$-profile, the internal inductance, and the poloidal beta are introduced. The properties of these quantities will be illustrated in the case of the tokamak, by applying the large aspect ratio tokamak approximation. The properties of a non-circular plasma cross-section and the role of the vertical field will also be discussed in this approximation. Some attention is given to the (numerical) problem of solving the equilibrium equation and of reconstructing a plasma equilibrium from external measurements. The numerical methods themselves are presented in [1].
\end{abstract}

\section{INTRODUCTION}

Magnetic confinement experiments in fusion research have a rich history in which a variety of magnetic configurations [2,3] have been tried, like stellarators, mirror machines and pinch experiments. The first considerations were to confine a plasma with as high as possible pressure in a magnetic field that is limited in terms of required currents and external forces. The confinement properties in many of these 'optimized' plasmas were below expectations due to instabilities. As the theoretical understanding of plasma instabilities grew, magnetic confinement devices that avoided these instabilities were designed. More refined devices would often be plagued by slightly more subtle instabilities. The tokamak emerged relatively late on the scene. Tokamaks are characterized by a strong toroidal magnetic field. However, as we will see, the force exerted by a purely toroidal field is not pointed towards the plasma in all points. It cannot prevent a toroidal plasma from expanding its major radius. Therefore a poloidal field is required as well. The poloidal field does not have to be as large as the toroidal field and in a typical tokamak it is considerably smaller, by a factor which scales with the aspect ratio (ratio of major radius to minor radius) of the device. A poloidal field inside the plasma is required for local force balance, and an external vertical field has to be added to prevent the plasma as a whole from increasing its major radius. The internal poloidal field can only be created by a large toroidal plasma current. This current is primarily created inductively, by the flux change in a transformer yoke through the torus. An important disadvantage of this scheme is that the flux can be changed only for a finite duration, so that stationary operation is not possible. A large research effort is presently being undertaken to drive a major fraction of the plasma current by means of injected radiofrequency waves and by the so-called bootstrap current, created during the rather complex loss processes that a high pressure tokamak plasma suffers. Another apparent flaw of the tokamak design is the wastefully large toroidal field, compared to the poloidal field and the plasma pressure it can confine. The important advantage of the tokamak is the inherent stability provided by both the strong toroidal field and the large plasma current. The immunity of a tokamak against some very fundamental and fast plasma instabilities can easily be shown, but it seems that many less straightforward plasma modes are naturally stabilized by the 'robustness' of this magnetic configuration.

In addition, some disruptive instabilities nowadays can be avoided thanks to the increased experimental experience with tokamak operation, and instabilities of the plasma position are tackled with improved magnetic control techniques, for instance. As a consequence, the most important instabilities that are persistent in modern tokamaks have a rather complicated spatial structure $[2,3,4]$ and are likely to involve subtle interactions between the kinetics of individual particles and collective plasma motion.

On short timescales a tokamak plasmas show a variety of oscillations and turbulent phenomena. On sufficiently long timescales (which depend on the size of the machine, resistivity of the plasma, etc.) the plasma behaviour is governed by gradual changes in the magnetic configuration (internal or imposed by the coils) and by changes of the plasma heating, the diffusive losses, and the ratios between particle species. We will consider situations where there exists an intermediate timescale on which the tokamak plasma is in "equilibrium". On this timescale, the plasma pressure and the magnetic forces must balance. The proper equations for this situation are provided by ideal MHD, which treats the plasma as an idealy conducting fluid, subject to the lowfrequency Maxwell equations (no displacement current in Ampère's law: $\boldsymbol{j}=\nabla \times \boldsymbol{B}$ ). Ideal MHD equilibria satisfy the force balance

$$
\nabla p=\boldsymbol{j} \times \boldsymbol{B} .
$$

Together with the constraints $\nabla \cdot \boldsymbol{B}=0$ and $\nabla \cdot \boldsymbol{j}=0$, this implies (according to a result from topology called Hopf's 
theorem) that in a volume where $\nabla p \neq 0$, the surfaces of constant pressure are tori (either simply nested or in braids), and one sees immediately from Eq. (1) that the field lines of $\boldsymbol{B}$ and $\boldsymbol{j}$ lie in these surfaces [5]. The field lines can either close in themselves after a finite number of revolutions, or fill a magnetic surface ergodically. The third possibility, that a field line fills a finite volume in space stochastically, can happen in ideal MHD only if $\nabla p=0$ (and indeed, in a less idealized plasma model, such a volume exhibits fast losses of particles and energy). These results are applicable to all closed confined plasma configurations and can even be generalized to cases with stationary plasma flow (rotation) or an anisotropic pressure tensor. In the following, we will restrict ourselves to magnetic fields that form a single set of nested toroidal flux surfaces around a circular magnetic axis.

From the above we can conclude that closed flux surfaces are required to support a pressure gradient $\nabla p \neq 0$, i.e., to confine the plasma. This is also what one expects after considering the microscopic picture, since individual particles stick to a given flux surface as long as they continue gyrating around the same field line.

The constraint $\nabla \cdot \boldsymbol{B}=0$ implies that on a given magnetic surface field lines cannot cross each other. Therefore, if one follows a field line around the torus, the ratio between the numbers of toroidal and poloidal revolutions of the field line converges to a constant $q$ for $n \rightarrow \infty$. The quantity $q$ is called the safety factor because of its importance in stability criteria for a wide range of MHD modes. It is related to the average pitch of the helical field on a magnetic surface. Specifying the $q$ values of all magnetic surfaces describes the complete topology of the magnetic field lines. In particular, the $q$ value of a magnetic surface with closed field lines is a rational number, since each field line closes after integer numbers of toroidal and poloidal revolutions.

\section{THE GRAD-SHAFRANOV EQUATION}

In order to describe axisymmetric MHD equilibria we will initially use the right-handed cylinder coordinates $(R, \phi, Z)$, where $\phi$ is the angle of symmetry and R measures the distance to the axis of symmetry (the major radius in a toroidal system). Using

$$
0=\nabla \cdot \boldsymbol{B}=\frac{1}{R} \frac{\partial}{\partial R}\left(R B_{R}\right)+\frac{1}{R} \frac{\partial B_{\phi}}{\partial \phi}+\frac{\partial B_{Z}}{\partial Z},
$$

and the symmetry $(\partial / \partial \phi=0$ when acting on a scalar) one can write $\boldsymbol{B}$ in terms of a stream function $\psi$,

$$
\boldsymbol{B}=\boldsymbol{B}_{p}+B_{t} \hat{\boldsymbol{\phi}} ; \quad \boldsymbol{B}_{p}=\nabla \psi \times \nabla \phi,
$$

where $\hat{\phi}=R \nabla \phi$ and where $B_{t}$ and $\psi$ do not depend on $\phi$. Whenever $\boldsymbol{B}_{p} \neq 0$, the magnetic field lines lie on surfaces of constant $\psi$. They are called flux surfaces because $\psi$ is related to the poloidal flux $\psi_{p}$, for instance through an annulus in the equatorial plane defined by

$$
S=\left\{Z=0, R\left(\psi_{1}\right)<R<R\left(\psi_{2}\right)\right\} .
$$

We write the poloidal field as $\boldsymbol{B}_{p}=\nabla \times(\psi \nabla \phi)$. The poloidal flux through the surface $S$ is

$$
\begin{aligned}
\psi_{p} & =\int_{S} \boldsymbol{B}_{p} \cdot d \boldsymbol{A}=\int_{S} \nabla \times(\psi \nabla \phi) \cdot d \boldsymbol{A} \\
& =\oint_{\partial S} \psi \nabla \phi \cdot d \boldsymbol{\ell}=2 \pi\left(\psi_{2}-\psi_{1}\right) .
\end{aligned}
$$

The total poloidal flux (vacuum as well as plasma field) through the circular magnetic axis is found by taking for $\psi_{1}$ and $\psi_{2}$ the values on the magnetic axis and at $R=0$ ), respectively.

The current density is given in terms of the magnetic field by Ampère's law,

$$
\begin{aligned}
\boldsymbol{j} & =\nabla \times \boldsymbol{B}=\nabla \times\left(\nabla \psi \times \nabla \phi+R B_{t} \nabla \phi\right) \\
& =-\triangle^{*} \psi \nabla \phi+\nabla\left(R B_{t}\right) \times \nabla \phi .
\end{aligned}
$$

where the elliptic, Laplacian-like Grad-Shafranov operator $\triangle^{*}$ is defined by

$$
\triangle^{*} \psi=R^{2} \nabla \cdot\left(\frac{\nabla \psi}{R^{2}}\right)=R \frac{\partial}{\partial R}\left(\frac{1}{R} \frac{\partial \psi}{\partial R}\right)+\frac{\partial^{2} \psi}{\partial Z^{2}} .
$$

(In the last step in (5) one can use the dyadic identity $R^{2} \nabla \nabla \phi=-\nabla R \nabla \phi-\nabla \phi \nabla R$.) The final ingredient is the momentum balance equation (1). We will discuss its three vector components formed by taking the scalar products of Eq. (1) with $\boldsymbol{B}, \boldsymbol{j}$, and $\nabla \psi$, respectively. The first component, $\boldsymbol{B} \cdot \nabla p=0$, implies that the flux surfaces are surfaces of constant $p(\psi)$ (" $p$ is a flux function"). Using this result, the second component of the force balance, $\boldsymbol{j} \cdot \nabla p=0$, tells that the current flows along the flux surfaces. For expression (5) it has the consequence that $R B_{t}$ is a surface quantity as well. We write

$$
R B_{t}=F(\psi) .
$$

This quantity $F(\psi)$ is related to the poloidal current through the surface $S$, given in Eq. (3). It follows from (7) that the poloidal current density, which is the second term in expression (5), can be written as $\boldsymbol{j}_{p}=\nabla \times(F \nabla \phi)$. Hence, the total current through $S$ can be derived as an integral similar to the one for the poloidal flux (4), is

$$
\begin{aligned}
I_{p} & =\int_{S} \boldsymbol{j}_{p} \cdot d \boldsymbol{A}=\int_{S} \nabla \times(F \nabla \phi) \cdot d \boldsymbol{A} \\
& =\oint_{\partial S} F \nabla \phi \cdot d \boldsymbol{\ell}=2 \pi\left(F\left(\psi_{2}\right)-F\left(\psi_{1}\right)\right) .
\end{aligned}
$$

If one takes $R\left(\psi_{1}\right)=0, I_{p}$ includes the current flowing in the toroidal field coils as well as the poloidal plasma current. Finally, in terms of the two flux functions $p(\psi)$ and $F(\psi)$, the force balance in the $\nabla \psi$ direction gives

$$
\triangle^{*} \psi=-R^{2} \frac{d p}{d \psi}-F \frac{d F}{d \psi} .
$$


This 2-dimensional partial differential equation in $\psi$ is called the Grad-Shafranov equation $[6,7,8]$. For given functions $p(\psi)$ and $F(\psi)$, it is an elliptic second order nonlinear differential equation for $\psi$. Sufficient boundary conditions would be to specify $\psi$ everywhere on a closed contour, by specifying the shape of one flux surface, for instance. If a fixed outer surface of the plasma is specified, then in essence the plasma-vacuum boundary is replaced by the surface of a perfect conductor (on which $\psi$ is necessarily constant). This is a fixed boundary condition, which defines $\psi$ in the entire plasma. By specifying a flux surface in the vacuum region, one has a free boundary problem. Taking into account the currents in the coils leads to a somewhat different approach [9]. One can use the known currents in the coils and an assumed plasma current distribution to compute $\psi$ on a boundary which is convenient for the computations, a rectangle, say. With these Dirichlet boundary conditions one then solves the Grad-Shafranov equation in the interior. This leads to a different plasma current distribution than originally assumed, and one iterates the procedure. As an alternative, or in addition to considering the coil currents and computing $\boldsymbol{B}$ in the metal parts as well as the vacuum, poloidal field measurements can be available close to the coils or near the plasma. This makes the set of boundary conditions altogether more inhomogeneous and a very adaptable equilibrium solver is required. The system can even be overdetermined and to a certain extent the functions $p(\psi)$ and $F(\psi)$ can then be computed.

\section{FLUX COORDINATES}

Of great importance in equilibrium calculations, but also in the modelling of plasma transport and in stability analyses, are flux coordinates $(r, \theta, \phi)$. Here $\phi$ is the usual toroidal angle. The radial coordinate $r(\psi)$ labels the flux surfaces. It can be the flux itself, or the volume enclosed by each flux surface, or can be chosen to closely resemble the minor radius (distance to the magnetic axis). One possibility is the minor radius at $Z=0$. Another definition takes the square root of the area of the cross section. The differences between such definitions are easy to account for and usually not very important. The various definitions $[10,11,12]$ used for the poloidal angle $\theta$, however, are convenient in very specific applications: ( $i$ ) the proper geometrical angle can be used when the geometry is fixed, for instance in tomographic diagnostic methods, (ii) an orthogonal coordinate system $(\nabla r \cdot \nabla \theta=0)$ is convenient in ballooning stability analysis and in solving the Grad-Shafranov equation by means of the conformal mapping method [13], (iii) and most universally applied, especially in stability studies, are coordinates in which the field lines appear straight [14]. In these coordinates the pitch $d \phi / d \theta$ of the field lines is a constant on each flux surface. This constant, of course, is precisely $q(\psi)$.

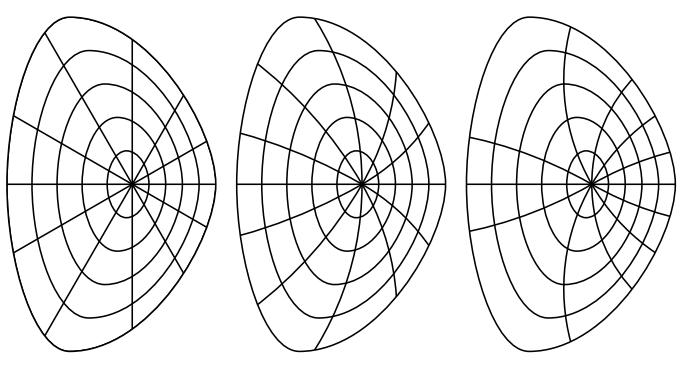

Figure 1: Flux coordinate systems. Left: proper poloidal angle; center: straight fieldline coordinates; right: orthogonal coordinates

For general flux coordinates $(r, \theta, \phi)$ the Jacobian is

$$
J \equiv|\nabla r \times \nabla \theta \cdot \nabla \phi|^{-1}=\frac{R}{|\nabla r|} \frac{d \ell}{d \theta} .
$$

The general line element and the metric tensor are now given by

$$
\begin{aligned}
& d s^{2}=g_{r r} d r^{2}+2 g_{r \theta} d r d \theta+g_{\theta \theta} d \theta^{2}+g_{\phi \phi} d \phi^{2}, \\
& g_{r r}=\frac{J^{2}}{R^{2}}|\nabla \theta|^{2}, \quad g_{r \theta}=-\frac{J^{2}}{R^{2}} \nabla \theta \cdot \nabla r, \\
& g_{\theta \theta}=\frac{J^{2}}{R^{2}}|\nabla r|^{2}, \quad g_{\phi \phi}=R^{2} .
\end{aligned}
$$

By applying the general operator identity

$$
\begin{aligned}
\nabla & =\nabla r \frac{\partial}{\partial r}+\nabla \theta \frac{\partial}{\partial \theta}+\nabla \phi \frac{\partial}{\partial \phi} \\
& =\frac{1}{J}\left(\frac{\partial}{\partial r} \nabla r+\frac{\partial}{\partial \theta} \nabla \theta+\frac{\partial}{\partial \phi} \nabla \phi\right) J
\end{aligned}
$$

to the operator $\triangle^{*}$, we can derive the Grad-Shafranov equation in flux coordinates,

$$
\frac{R^{2}}{J} \psi^{\prime}\left[\frac{\partial}{\partial r}\left(\frac{g_{\theta \theta} \psi^{\prime}}{J}\right)-\frac{\partial}{\partial \theta}\left(\frac{g_{r \theta}}{J}\right) \psi^{\prime}\right]+R^{2} p^{\prime}+F F^{\prime}=0,
$$

where a prime denotes $d / d r$. This expression can immediately be adapted to any particular flux coordinate system. In particular, we will use it in the next section, where we will employ approximate flux coordinates to describe flux surfaces with almost circular poloidal cross sections.

We will now use the coordinate system to express the safety factor $q(\psi)$ in terms of the magnetic field. Due to toroidal symmetry, the ratio between toroidal and poloidal revolutions of the field lines can be found with an integral of $d \phi / d \theta$ over just one poloidal revolution,

$$
q(\psi)=\frac{1}{2 \pi} \int_{0}^{2 \pi} \frac{d \phi}{d \theta} d \theta=\frac{1}{2 \pi} \oint \frac{B_{t}}{R} \frac{d \ell}{B_{p}} .
$$

Here we have used that the pitch of the field lines is locally given by the ratio between the toroidal and poloidal field 
components. By writing $\boldsymbol{B}=\nabla \psi \times \nabla \phi+F(\psi) \nabla \phi$ the safety factor can be written as

$$
q(\psi)=\frac{F}{2 \pi} \oint \frac{d \ell}{R|\nabla \psi|}=\frac{F}{2 \pi \psi^{\prime}} \oint \frac{J}{R^{2}} d \theta .
$$

The last definition we introduce here in general flux coordinates is the flux surface averaged value $\langle X\rangle$ of a given quantity $X$. In order to be compatible with volume integrals, $\int\langle X\rangle d^{3} x=\int X d^{3} x$, it is a suitably weighed integral over the flux surface,

$\langle X\rangle=\lim _{\Delta V \rightarrow 0} \frac{1}{\Delta V} \int_{\psi}^{\psi+\Delta \psi} X d V=\frac{\oint X \frac{d \ell}{B_{p}}}{\oint \frac{d \ell}{B_{p}}}=\frac{\int_{0}^{2 \pi} X J d \theta}{\int_{0}^{2 \pi} J d \theta}$.

Here, $V(\psi)$ is the volume enclosed by the flux surface given by $\psi$. The integral in (10) can be written as a flux average,

$$
q=\frac{F}{4 \pi^{2}}\left\langle\frac{1}{R^{2}}\right\rangle \frac{d V}{d \psi} .
$$

\section{LARGE ASPECT RATIO EXPANSION}

All tokamak equilibria have some global properties in common, which can be discussed either qualitatively or quantitatively, but in the latter case this generally requires numerical solutions of the Grad-Shafranov equation. Instead, we will restrict ourselves to a discussion of equilibria with almost circular poloidal cross sections and a small inverse aspect ratio $\varepsilon$, so that a purely analytic treatment is possible $[2,10,11]$. Thus we will discuss some properties of plasma shaping effects (deviations from circular cross sections) and the outward shift, called Shafranov shift), of the plasma centre under the influence of high plasma pressure.

Our flux coordinates will be $\theta$, the geometric angle with respect to the Shafranov shifted [10] center of each flux surface $(R, Z)=\left(R_{0}-\Delta(\psi), 0\right)$, and $r(\psi)$, the $\theta$-averaged minor radius of the flux surface. The distance $\rho$ to the centre of a flux surface will be subject to shaping functions $S_{m}(r)$ $(m \geq 2)$,

$$
\begin{aligned}
R & =R_{0}-\Delta(r)+\rho \cos \theta \\
Z & =\rho \sin \theta \\
\rho & =r-\sum_{m \geq 2} S_{m}(r) \cos m \theta .
\end{aligned}
$$

The relation to the generally used notation

$$
\begin{aligned}
& R=R_{0}+a \cos (\theta-\delta \sin 2 \theta) \\
& Z=\kappa a \sin \theta
\end{aligned}
$$

is that the elongation is $\kappa=1+2 S_{2} / r$ and the triangularity is $\delta=4 S_{3} / r$. Our approximation is $\varepsilon=a / R_{0} \ll 1$; hence we use the ordering $r=\mathcal{O}(\varepsilon R)$. Deviations from centered circles are taken to be one order smaller, $\Delta=\mathcal{O}\left(\varepsilon^{2} R\right)$, and
$S_{m}=\mathcal{O}\left(\varepsilon^{2} R\right)$. We will neglect smaller terms, $\mathcal{O}\left(\varepsilon^{3} R\right)$, throughout. In order to express the Grad-Shafranov equation in these coordinates we need

$$
\begin{aligned}
g_{r \theta} & =\frac{\partial Z}{\partial r} \frac{\partial Z}{\partial \theta}+\frac{\partial R}{\partial r} \frac{\partial R}{\partial \theta} \\
& =r \Delta^{\prime} \sin \theta+\sum_{m} m S_{m} \sin m \theta+\mathcal{O}\left(\varepsilon^{2} r\right), \\
g_{\theta \theta} & =\left(\frac{\partial Z}{\partial \theta}\right)^{2}+\left(\frac{\partial R}{\partial \theta}\right)^{2} \\
& =r^{2}-2 r \sum_{m} S_{m} \cos m \theta+\mathcal{O}\left(\varepsilon^{2} r^{2}\right), \\
J & =R\left(\frac{\partial R}{\partial \theta} \frac{\partial Z}{\partial r}-\frac{\partial R}{\partial r} \frac{\partial Z}{\partial \theta}\right) \\
& =-r R_{0}\left[1+\left(\frac{r}{R_{0}}-\Delta^{\prime}\right) \cos \theta\right. \\
& \left.\quad-\sum_{m}\left(S_{m}^{\prime}+\frac{S_{m}}{r}\right) \cos m \theta+\mathcal{O}\left(\varepsilon^{2}\right)\right] .
\end{aligned}
$$

Substituting these expressions in Eq. (9), and keeping only leading order and $\mathcal{O}(\varepsilon)$ terms in the result, we obtain

$$
\begin{aligned}
& \frac{\psi^{\prime 2}}{r}\left\{1+\left(r \Delta^{\prime \prime}+\Delta^{\prime}-\frac{r}{R_{0}}\right) \cos \theta\right. \\
& \left.\quad+\sum_{m}\left[r^{2} S_{m}^{\prime \prime}+r S_{m}^{\prime}+\left(1-m^{2}\right) S_{m}\right] \cos m \theta\right\} \\
& +\psi^{\prime} \psi^{\prime \prime}\left\{1+2 \Delta^{\prime} \cos \theta+2 r \sum_{m} S_{m}^{\prime} \cos m \theta\right\} \\
& +R_{0}^{2}\left(1+2 \frac{r}{R_{0}} \cos \theta\right) p^{\prime}+F F^{\prime}=0 .
\end{aligned}
$$

The leading order terms do not depend on $\theta$ and form the radial force balance,

$$
\frac{1}{2 r^{2}}\left(r^{2} \psi^{\prime 2}\right)^{\prime}+R_{0}^{2} p^{\prime}+F F^{\prime}=0 .
$$

It is the balance between the forces exerted by the plasma pressure $(\nabla p)$ and by the magnetic pressure $\left(\nabla \frac{1}{2} B^{2}\right)$, and describes how the poloidal and toroidal magnetic fields prevent the plasma from expanding in the $\nabla r$ direction. It would be the exact force balance if the plasma column were a cylinder. We will define now the quantity poloidal beta, in an arbitrary flux surface enclosing a volume $V_{0}$ as

$$
\beta_{p}\left(r_{0}\right)=\frac{2}{V_{0}} \frac{\int_{0}^{V_{0}} p d V}{\left\langle B_{p}^{2}\right\rangle_{0}},
$$

In the case of circular cross sections this becomes

$$
\beta_{p}\left(r_{0}\right)=-2 \frac{R_{0}^{4} q^{2}}{F^{2} r_{0}^{4}} \int_{0}^{r_{0}} p^{\prime} r^{2} d r .
$$

Multiplication of Eq. (12) by $r^{2}$ and integrating yields

$$
\beta_{p}\left(r_{0}\right)=1+\frac{1}{r_{0}^{2} \psi^{\prime 2}} \int_{0}^{r_{0}} \frac{d F^{2}}{d r} r^{2} d r .
$$


One sees that if $\beta_{p}>1$, then $d F^{2} / d r>0$ and the toroidal field contributes to confinement of the pressure. If $\beta_{p}<1$, however, the toroidal field counters confinement since then $d F^{2} / d r<0$. However, due to the toroidal shape of the configuration, radial confinement is not sufficient, since $\theta$ dependent terms unavoidably appear in the force balance equation. They are the factors $R$ that appear in the GradShafranov equation (10) and the operator (6). Consequently, Eq. (11) contains $\mathcal{O}(\varepsilon)$ terms proportional to $\cos \theta$. This component of the Grad-Shafranov equation yields a radial equation for the Shafranov shift [10],

$$
\Delta^{\prime \prime}+\left(2 \frac{\psi^{\prime \prime}}{\psi^{\prime}}+\frac{1}{r}\right) \Delta^{\prime}-\frac{1}{R_{0}}+2 \frac{r R_{0} p^{\prime}}{\psi^{\prime 2}}=0 .
$$

This equation expresses how the poloidal field prevents the flux surfaces from expanding in the $\nabla R$ direction. Since the shift $\Delta$ is always smaller than the plasma minor radius, this equation limits the pressure to values of the order of $R B_{p}^{2} / a$, which for $B_{p} \sim \varepsilon B_{t}$ implies that the pressure is $\mathcal{O}(\varepsilon)$ smaller than the magnetic pressure,

$$
\beta=\frac{2 p}{B^{2}}=\mathcal{O}(\varepsilon),
$$

which is called the high- $\beta$ tokamak ordering. In our ordering, $\Delta^{\prime}=\mathcal{O}(\varepsilon r)$, which corresponds to the low- $\beta$ tokamak ordering,

$$
\beta=\frac{2 p}{B^{2}}=\mathcal{O}\left(\varepsilon^{2}\right)
$$

Equation (14) can be integrated after writing $\psi^{\prime}=$ $-r F / q R_{0}+\mathcal{O}\left(\varepsilon^{2}\right)$ and multiplying by $r^{3} / q^{2}$. This yields

$$
\Delta^{\prime}=\frac{r}{R_{0}}\left(\beta_{p}+\frac{1}{2} \ell_{i}\right),
$$

where the normalized internal inductance at a flux surface with $r=r_{0}$ and enclosed volume $V_{0}$ is defined by

$$
\ell_{i}\left(r_{0}\right)=\frac{2 L_{i}}{R_{0}}=\frac{4}{I_{p}^{2}\left(r_{0}\right) R_{0}} \int_{0}^{V_{0}} \frac{1}{2} B_{p}^{2} d V .
$$

The plasma internal and external inductances are given by

$$
\frac{1}{2} L_{i} I_{p}^{2}=\int_{\text {plasma }} \frac{1}{2} B_{p}^{2} d V, \quad \frac{1}{2} L_{e} I_{p}^{2}=\int_{\text {plasma }} \frac{1}{2} B_{p}^{2} d V .
$$

In the case of a circular cross section Eq. (16) gives approximately

$$
\ell_{i}\left(r_{0}\right)=2 \frac{q^{2}}{r_{0}^{4}} \int_{0}^{r_{0}} \frac{r^{3}}{q^{2}} d r .
$$

Equation (15) describes force balance in the $\nabla R$ direction in the interior of the plasma. The first term to the right arises because the plasma pressure tries to increase the plasma volume. Since $V \approx 4 \pi^{2} r_{0}^{2} R$, this can be done not only by increasing the minor radius of a flux surface $\left(p^{\prime}\right.$-term in $\mathrm{Eq}(12))$ but also by increasing its major radius. The second term on the right is due to the toroidal current. It is the hoop force due to the self-inductance of a current carrying circuit. The balancing term on the left is caused by the compression of the flux surfaces, i.e., compression of the poloidal flux, at the low field side of the torus.

At the plasma surface $r=a$, the horizontal force balance is not automatically satisfied. At the plasma surface the poloidal field is

$$
\begin{aligned}
B_{p} & =\frac{|\nabla \psi|}{R}=\frac{\psi^{\prime} g_{\theta \theta}^{1 / 2}}{J} \\
& =-\frac{\psi^{\prime}}{R_{0}}\left[1+\frac{a}{R_{0}}\left(\beta_{p}+\frac{1}{2} \ell_{i}-1\right) \cos \theta\right] .
\end{aligned}
$$

These values of $B_{p}$ on the surface should match the solution in the surrounding vacuum region. The vacuum field satisfies $\nabla \times \boldsymbol{B}=0$, i.e., $\triangle^{*} \psi=0$. It is possible to integrate the vacuum equation outward starting with the initial values (17). The dominant contribution in (17), the poloidal field generated by the plasma current, vanishes for $R \rightarrow \infty$. The $\cos \theta$ terms in (17) and in $\triangle^{*}$ do not vanish at infinity, however. The asymptotic value of $B_{p}$ is proportional to $\cos \theta$, i.e., it is a vertical field. In other words, the surface poloidal field (17) cannot be generated by the plasma current alone. An external vertical field is required. This field can be provided by vertical field coils, but for short tokamak discharges a conducting shell sufficiently close to the plasma can provide the force balance. The poloidal flux is then compressed between the plasma and the conducting shell when the plasma shifts outward during heating. In practical situations the calculation of the external fields can be complicated, but by computing the integrated quantity $\left\langle B_{p} \cos \theta\right\rangle$ from Eq. (17) one can arrive after some manipulations at the following identity given by Freidberg [2],

$2 \pi R_{0} I_{p} B_{v}=\frac{1}{2} I_{p}^{2} \frac{\partial}{\partial R_{0}}\left(L_{e}+L_{i}\right)-2 \pi^{2} \int d r r^{2}\left(p^{\prime}-\frac{F F^{\prime}}{R_{0}^{2}}\right)$.

The term on the left-hand side is the inward force of the vertical field acting on the toroidal plasma current. It balances the four outward forces on the right. The first two of these represent the hoop force of the plasma current via the selfinductance, to which the poloidal fluxes inside and outside the plasma contribute. The third term is the tire tube force due to the pressure gradient, and the last term is the $(1 / R)$ magnetic force, directed outward if $\beta_{p}<1$ and inward if $\beta_{p}>1$ (see comments below Eq. (13)).

Equation (17) also expresses that the quantity $\beta_{p}+\frac{1}{2} \ell_{i}$ of the entire plasma can be deduced from the external poloidal field, without knowing the pressure and current distribution in the plasma. However, this measurement alone does not provide the total plasma pressure (as expressed in $\beta_{p}$ ) separately.

More information can be extracted from the vacuum field if the plasma has a non-circular cross section. Equation (11) contains the higher poloidal harmonics due to shap- 
ing coefficients. The $\cos m \theta$ terms are

$$
S_{m}^{\prime \prime}+\left(2 \frac{\psi^{\prime \prime}}{\psi^{\prime}}+\frac{1}{r}\right) S_{m}^{\prime}+\frac{1-m^{2}}{r^{2}} S_{m}=0 .
$$

Near the plasma centre, $\psi^{\prime}$ is approximately proportional to $r$ so that this equation becomes

$$
S_{m}^{\prime \prime}+\frac{3}{r} S_{m}^{\prime}+\frac{1-m^{2}}{r^{2}} S_{m}=0,
$$

which has solutions $S_{m} \sim r^{ \pm m-1}$. Only the solution $\sim r^{m-1}$ is regular in $r=0$. If one integrates Eq. (18) outward, starting with the regular solution at $r=0$, one obtains $S_{m}$ and $S_{m}^{\prime}$ at the plasma boundary. These values can be determined by external measurements. Since equation (18) involves $\psi^{\prime} \sim r / q$, these measurements provide (crude) information about the $q(r)$ profile. This gives an estimate of $\ell_{i}$. Concluding, $\beta_{p}$ can be obtained directly from magnetic measurements if there is sufficient plasma shaping, especially elongation.

\section{EQUILIBRIUM IDENTIFICATION}

For several purposes it is required to know the equilibrium configurations of tokamak plasmas, i.e., the current profile, the pressure profile, and the positions and shapes of the flux surfaces (or, alternatively, the direction and magnitude of the poloidal magnetic field). Primarily, this information has to be deduced from the known coil currents and magnetic measurements well outside the plasma, typically near the coils. The problem is to find a smooth solution of the GradShafranov equation that best fits the measurements (Neumann conditions on a closed boundary) [9]. Smoothing, and the inclusion of assumptions on "reasonable" profiles is necessary because the reconstruction problem is inherently illposed: small changes (errors, inconsistencies) in the magnetic measurements outside the plasma cause changes in the solution that inflate dramatically further inward.

When accuracy rather than speed and automatic operation is the issue, a lot can be gained by using additional information: density and temperature measurements provide information on the pressure profile, motional Stark effect or Faraday rotation give poloidal field values inside the plasma, and hence information about the current density. In addition there can be discrete pieces of information: is the plasma touching the limiter? Do observed plasma oscillations give the radius of a specific rational- $q$ surface?

Much depends on the specific purpose of the reconstruction. Here we list a few.

1. Fast reconstruction during the discharge. During a discharge, immediate knowledge about the plasma position is often required for control. This requires good modelling of the vacuum boundary conditions, the coils, and the iron around the machine. The modelling of the plasma equilibrium itself need not be as accurate as in other applications.
Therefore crude assumptions about the plasma current and pressure profiles may be used.

2. Interpretation of some plasma measurements. Some tokamak diagnostics measure quantities inside the plasma integrated over a line of sight. If the original quantity is a flux function (for instance the electron density as measured by an interferometer system), additional information is required to deduce this flux function from the set of line integrated measurements. This is required for instance if one has to combine different flux quantities (density and temperature in order to obtain the pressure). For such a profile reconstruction one needs to know both the positions of the lines of sight and the shapes and positions of the flux surfaces. Since the problem is entirely geometrical, accuracy of the pressure and current profiles in the Grad-Shafranov equation is only important insofar the shapes of the flux surfaces are concerned.

3. Plasma energy, heating and transport simulations. To study transport rates and local power balance in the plasma it is important to get optimal information about the plasma pressure from magnetic and other measurements. For correct interpretation of the data, the presence of impurity particle species and non-thermal particle populations may have to be accounted for.

4. Stability analysis. In order to analyze the stability of plasmas (planned or experimentally produced), knowledge of the current density (q-profile) and local pressure gradients is important. For many numerical stability calculations, a precise numerical solution of the Grad-Shafranov-equation is more important than an accurate fit to all available experimental data.

\section{ACKNOWLEDGMENT}

This work, supported by the European Communities under the contract of Association between EURATOM/FOM, was carried out within the framework of the European Fusion Programme with financial support from NWO. The views and opinions expressed herein do not necessarily reflect those of the European Commission.

\section{REFERENCES}

1. R. Keppens and J.W.S. Blokland, "Computing ideal magnetohydrodynamic equilibria", these proceedings.

2. J.P. Freidberg, "Ideal Magnetohydrodynamics", Plenum Press, New York (1987).

3. J.P. Freidberg, "Ideal magnetohydrodynamic theory of magnetic fusion systems," Rev. Mod. Phys. 54, 801 (1982).

4. H.J. de Blank, "MHD instabilities in tokamaks", these proceedings.

5. M.D. Kruskal and R.M. Kulsrud, "Equilibrium of a magnetically confined plasma in a toroid," Phys. Fluids 1, 265 (1958). 
6. H. Grad and H. Rubin, "Hydromagnetic equilibria and force-free fields," in "Proceedings of the Second United Nations International Conference on the Peaceful Uses of Atomic Energy" United Nations, Geneva, Vol. 32, p. 42 (1958).

7. R. Lüst and A. Schlüter, "Axialsymmetrische magnetohydrodynamische Gleichgewichtskonfigurationen", $Z$. Naturforschung. 12A, 850 (1957).

8. V.D. Shafranov, "On magnetohydrodynamical equilibrium configurations," Zhurnal Experimentalnoi i Teoreticheskoi Fiziki 33 (1957) 710 [Sov. Phys. J.E.T.P. 6 (1958) 545].

9. T. Takeda and S. Tokuda, "Computation of MHD equilibrium of tokamak plasma" Journ. Comp. Phys. 93, 1 (1991).

10. V.D. Shafranov, "Plasma equilibrium in a magnetic field," in "Reviews of Plasma Physics" (edited by M.A. Leontovich), Consultants Bureau, New York, Vol. 2, p. 103 (1966).

11. L.S. Solov'ev and V.D. Shafranov, "Plasma confinement in closed magnetic systems," in "Reviews of Plasma Physics" (edited by M.A. Leontovich), Consultants Bureau, New York, Vol. 5, p. 1 (1970).

12. L.E. Zakharov and V.D. Shafranov, "Equilibrium of current-carrying plasmas in toroidal configurations," in Reviews of Plasma Physics (edited by M.A. Leontovich), Consultants Bureau, New York, Vol. 11, p. 153 (1986).

13. J.P. Goedbloed, "Conformal mapping methods in twodimensional magnetohydrodynamics," Comput. Phys. Commun. 24, 311 (1981).

14. S. Hamada, "Hydromagnetic equilibria and their proper coordinates," Nucl. Fusion 1, 23 (1962). 